\title{
Evaluation in Vivo of the Accuracy of Different Impression Materials
}

\author{
Msc. Kreshnik Keraj* \\ Faculty of Dental Medicine, Tirana, Albania \\ *Corresponding author: kkeraj@yahoo.com
}

Received April 30, 2014; Revised May 13, 2014; Accepted May 19, 2014

\begin{abstract}
All prosthetic treatments are characterized by a sequence of well-structured clinical and laboratory steps, during which different kinds of impressions are required. The accuracy of an impression material plays an important part in the production of a well-fitting restoration and for the longevity of the restoration too. The aim of this study is to evaluate and to compare the accuracy of different impression materials, their ability to reproduce details, using a measuring microscope. Four types of impression materials were used to take the impression of the same reference points on the composite filling in the second premolar (35). Irreversible hydrocolloids: Orthoprint and Neocolloid (ZHermack), the Polyvinilsilosan: Elite HD+ and the Polyether: Permdine Garant (3M ESPEE) were used. The results show that all impression materials demonstrated greater characteristics if treated correctly before and after the impression is taken, in conformity with the respective manufacturers' guide lines and in respect to the storage time, so alginate if it's pouring into 1 hour, polyether into 4 days and polyvinilsiloxan into 2 weeks. So, we conclude that the quality and accuracy of the impression is affected by pouring and storage time in order to obtain a greater accuracy of stone casts.
\end{abstract}

Keywords: impression materials, accuracy, storage time

Cite This Article: Msc. Kreshnik Keraj, "Evaluation in Vivo of the Accuracy of Different Impression Materials." International Journal of Dental Sciences and Research, vol. 2, no. 3 (2014): 55-58. doi: 10.12691/ijdsr-2-3-2.

\section{Introduction}

Various types of impression materials are used in fixed and removable prosthodontics for the reproduction of oral structures. This in vivo investigation evaluates the accuracy of alginate, polyether and polysiloxane materials, measuring with microscope their ability to reproduce details. Analysis of variance ANOVA and analysis of comparisson Bonferroni were used to compare the accuracy of alginate, PE and PVS.

The objectives of this study are:

1. To evaluate the ability of each impression material to reproduce the details of the master

2. To compare the accuracy of those four impression materials with each other

3. To evaluate their dimensional stability affected by the time

The four impression materials investigated in the present study are:

1. Permadyne Garant (3M Espee, Seefeld-Germany).

2. Orthoprint Alginate (Zhermack, Badia Polesine, Rovigo-Italy).

3. Neocolloid Alginate (Zhermack, Badia Polesine, Rovigo-Italy).

4. Elite HD+ Light Body (Zhermack, Badia Polesine, Rovigo-Italy) Figure 1.

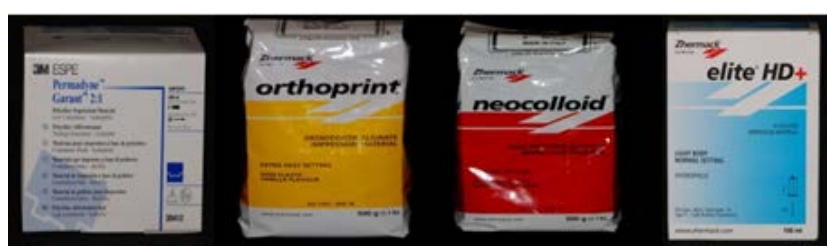

Figure 1. Impression materials

\section{Material and Methods}

In order for being $100 \%$ clinically realistic, all the impressions are taken in vivo conditions. We prepared an occlusal composite filling on a second premolar of mandible (35) like a master model for obtaining the impressions of the same referent points with different impression materials (Figure 2).

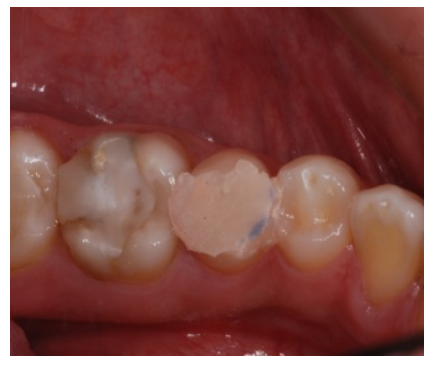

Figure 2. The composite filling on a second premolar 
A commonly file was placed on the occlusal surface of second premolar, next the flow composite was applied and a celluloid strip over the composite to create a smooth surface, than it is polymerised. (Figure 3)

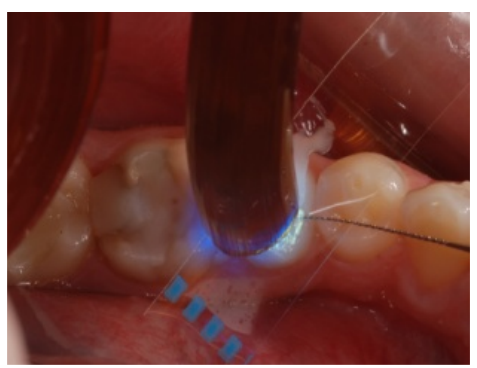

Figure 3. Polymerization

After that we removed the file with the composite filling too, and out of the mouth, we made a triangle on the celluloid strip, after that we removed the strip to obtain the triangle on the composite surface in which are the three segments to be measured(AB, BC and $A C)$ Figure 4.

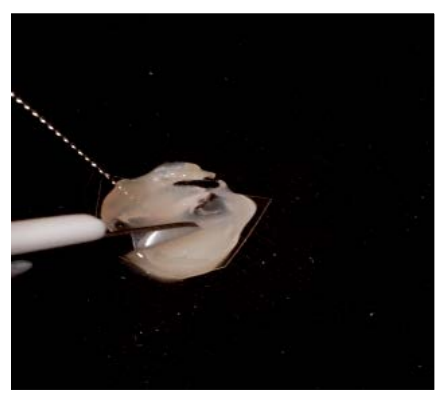

Figure 4. Execution of points of reference.

To measure the three segments on the master model we used Zeiss Microscop of the following parameters $(1.6 \times 2457 \mu$ per pixel) Figure 5 .

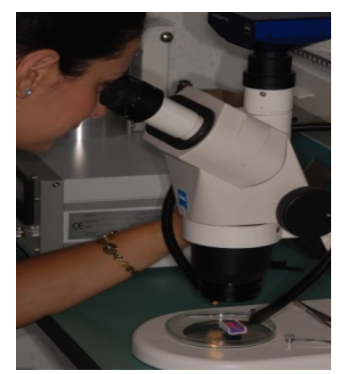

Figure 5. Zeiss (Axio Cam MRc 1.6×2457micromet/pixel

The view of Master Model seen on Microscope (Figure $6)$.

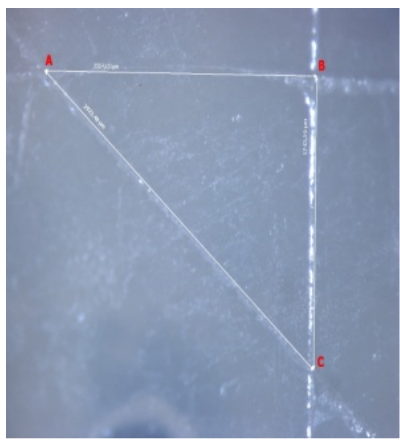

Figure 6. Master Model.
These are the lengths being measured several times. The bottom data of the following table represent the average of each length being measured (Table 1).

Table 1. Measure of Model Master

\begin{tabular}{|c|c|c|}
\hline $\mathrm{AB} \mu$ & $\mathrm{BC} \mu$ & $\mathrm{AC} \mu$ \\
\hline 2354.03 & 1742.19 & 2923.48 \\
\hline 2341.26 & 1735,41 & 2902.83 \\
\hline 2372.44 & 1805.61 & 2909.55 \\
\hline 2369.42 & 1792.26 & 2899.79 \\
\hline 2370.35 & 1799.72 & 2893.05 \\
\hline 2370.35 & 1799.72 & 2893.05 \\
\hline
\end{tabular}

After that we fixed another time the composite filling in the occlusal surface of premolar (with super attack) and we are ready to continue with the impressions taken, in vivo conditions, (Figure 7)

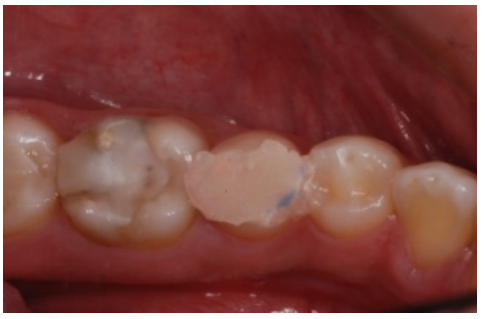

Figure 7. The model master fixed on the second premolar.

For this experiment we prepared 44 trays and we used the respective adhesive for each impression material. All the trays are of the same size and the impressions are taken from the same doctor on the same referent points (on the triangular in the composite filling).

We started with Polyether for taking the impression with the appropriate equipment.

We put the material with the Kerr pistol on the occlusal surface while at the same time the assistant fills the tray with Pentamix 2 and we put it in the mouth. (Figure 8) we obtained 11 impressions of the Model Master with PE. All these impressions are measured doing 11 measurements for each length on the microscope with the same zoom. (Figure 9)

The measurements are made after 24 hours, 4 days and 1 week to see if these materials change dimension.

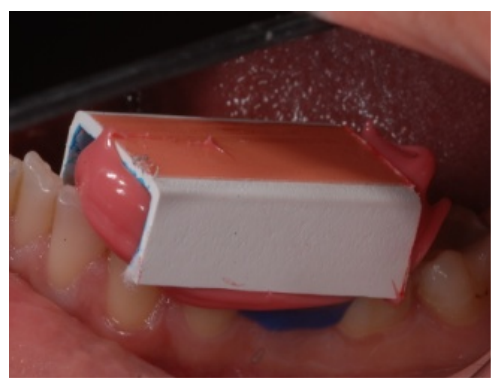

Figure 8. Taking impression with $\mathrm{PE}$

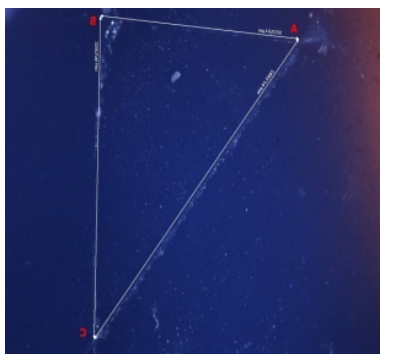

Figure 9. Polyether seen in Microscope 
All these data taken from PE measured after 24 hours, 4 days, 1 week, were processed through the statistic program Anova in order to have the result analysis. Where if the $\mathrm{P}<0.5$ that indicate statistical difference the changes are significant.

\begin{tabular}{|c|c|c|c|c|c|}
\hline \multicolumn{6}{|c|}{--- ANOVA - Analysis of Variance --- } \\
\hline Group & $\mathrm{N}$ & Mean & \multicolumn{2}{|c|}{ Std Dev } & SEM \\
\hline 1 & 5 & 2361 & \multicolumn{2}{|c|}{13.38} & 5.983 \\
\hline 2 & 5 & 2377 & \multicolumn{2}{|c|}{22.86} & 10.22 \\
\hline 3 & 5 & 2477 & \multicolumn{2}{|c|}{13.9} & 6.215 \\
\hline 4 & 5 & 2538 & \multicolumn{2}{|c|}{43.96} & 19.66 \\
\hline \multicolumn{6}{|c|}{$\mathrm{F}=50.02 \mathrm{P}=0.000$} \\
\hline \multicolumn{6}{|c|}{--- Multiple Comparisons - Bonferroni --- } \\
\hline Comparison & & \multicolumn{2}{|c|}{ Difference of means } & $\mathrm{t}$ & $\mathrm{P}<.05$ \\
\hline 2 vs. $1:$ & & \multicolumn{2}{|c|}{$2377-2361=15.8$} & 0.940 & No \\
\hline 3 vs. 1: & & \multicolumn{2}{|c|}{$2477-2361=116.2$} & 6.911 & Yes \\
\hline 4 vs. $1:$ & & \multicolumn{2}{|c|}{$2538-2361=177$} & 10.527 & Yes \\
\hline
\end{tabular}

We follow the same procedure for alginate and PVS.

Impressions were taken with PVS [1] with the Kerr pistols applied on the tray (Figure 10).

We obtained 11 impressions.

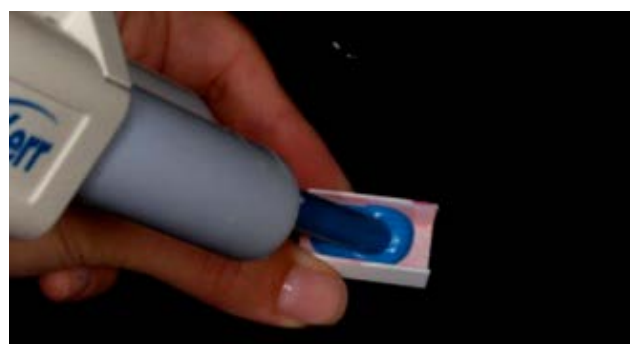

Figure 10. Taking Impression with PVS

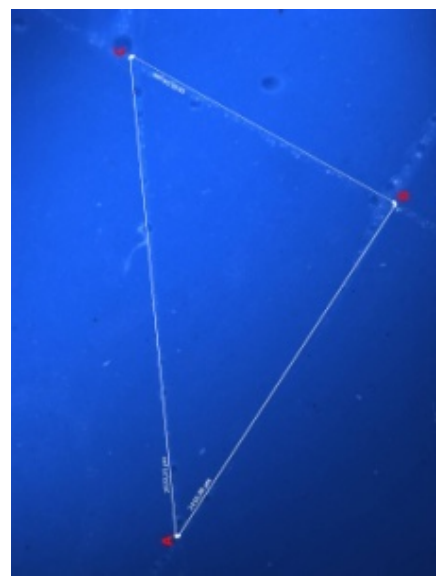

Figure 11. Elite HD+Seen on the Microscope

Than we passed on to taking the impression with Alginate using the mixer Microna, using the respective adhesive too (Figure 12).

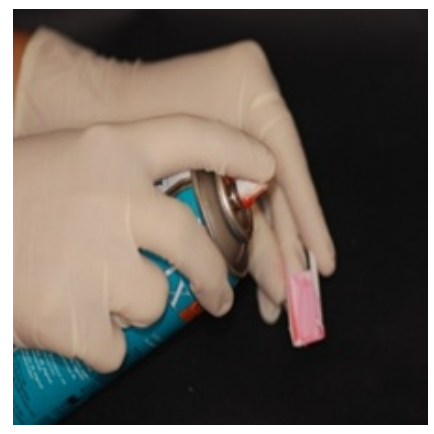

Figure 12. Alginate adhesive.
We used the appropriate proportion water/powder following the indication provided by the production company. We set the time 22 seconds and we obtain a very homogeneous mass without bubbles [3] (Figure 13).

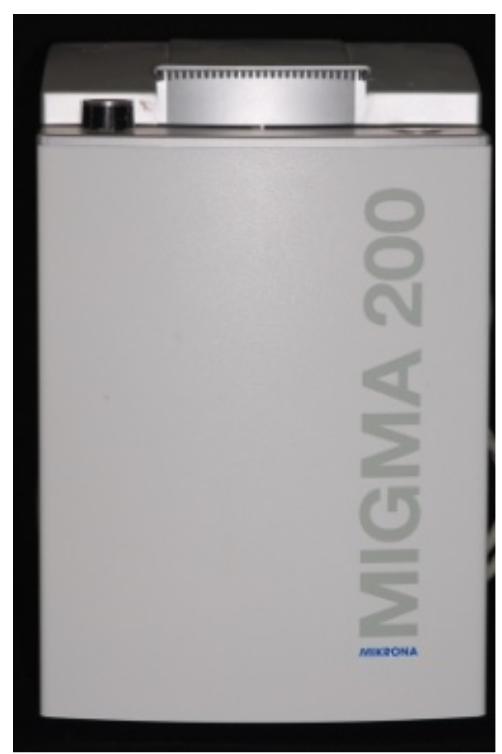

Figure 13. Mixer Alginate (Microna)

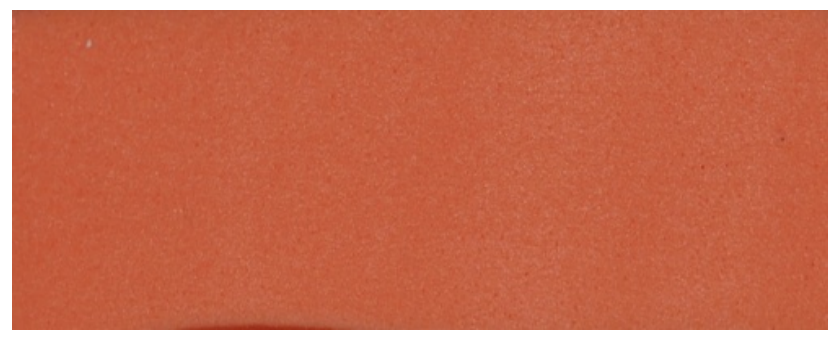

Figure 14. Alginate made by Microna, seen on the Microscope

We applied the Alginate on the tray and take the impression. We take 11 impressions for each Alginate (Figure 15).

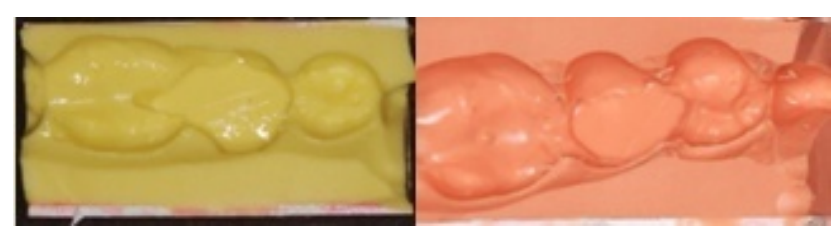

Figure 15. Orthoprint Neocolloid

Views of each Alginate seen on the Microscope (Figure 16):

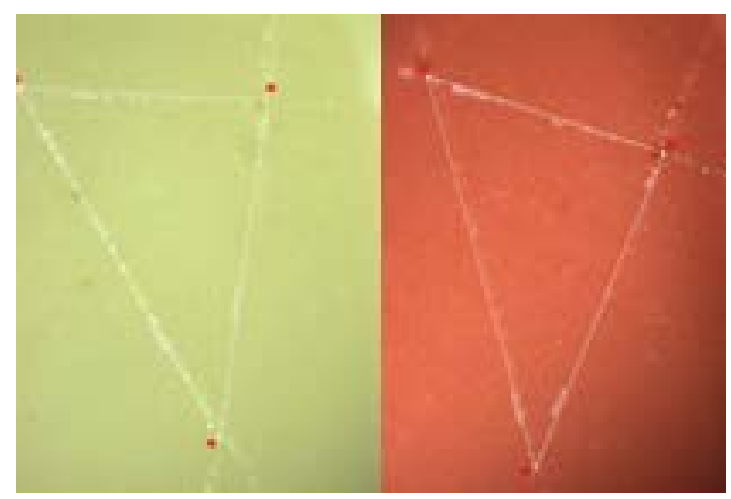

Figure 16. Orthoprint Neocolloid 
Following the measurements on the microscope for each material, we put all the data with the data of the Model Master on the Anova program to see the differences between them for each length $A B, B C, A C$.

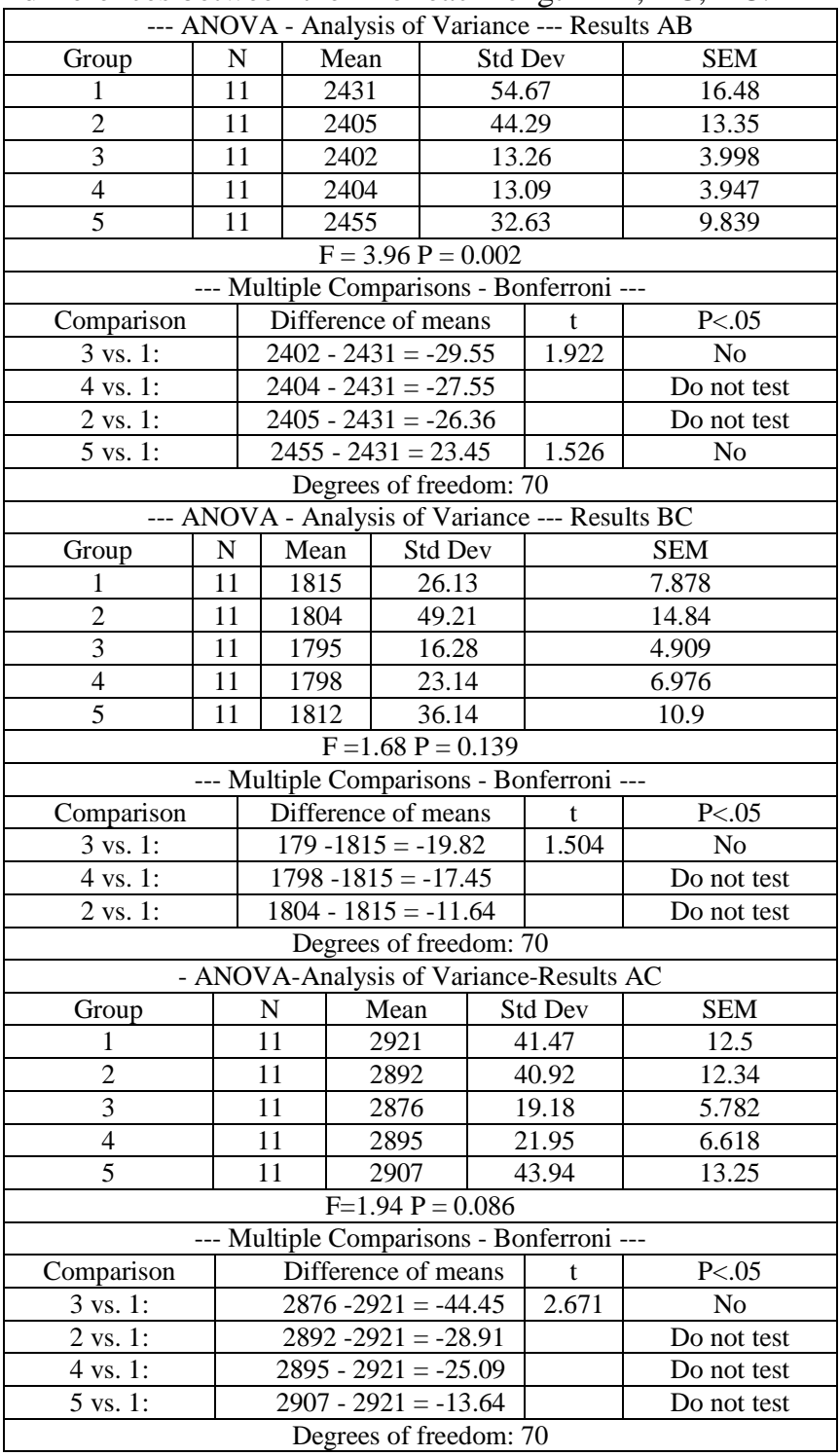

\section{Results}

The differences between the Master Model and the Polyether measured after 24 hours and 4 days were insignificant $(p<0.00)$. Since after 4 days or a week differences begin to be significant, so PE loose the dimensional stability.

The differences between the Master Model and Alginate measured into the first hour were insignificant too $(p=0.002)$.Since after this time the differences begin to be significant.

The differences between the Master Model and PVS (Elite HD+) measured after 4 days and 1 week was insignificant. They were insignificant measured after 2 weeks too.

\section{Discussion}

All impression materials demonstrated greater accuracy reproducing the master model. The alginate impressions measured into the first hour demonstrated the same accuracy of polyether and polyvinilsiloxane. The four impression materials had the same accuracy, with the minimum of differences 20-40 $\mu \mathrm{m}$ that's result insignificant for us. But alginate has the disadvantage of pouring time.

\section{Conclusions}

This study was an in vivo evaluation of the accuracy of four impression materials.

Based on the data obtained by the statistical program Anova we reached to the conclusion that Polyether, PVS and Alginate are comparable between them. They demonstrate insignificant statistical differences if we respect the pouring time: Alginate if poured within the first hour, Polyether within 4 days and PVS within 2 weeks.

\section{References}

[1] Di Felicia R, Scotti R, Belser UC. The influence of the mixing technique on the content of voids in two polyether impression materials. Schweiz Monatsschr Zahnmed, 2002; 112: 12-16.

[2] Kotwal K. Mixing bubble-free smooth alginate. Journal of Prosthetic Dentistry, 1984; 52:902.

[3] Shelley JJ, Alsup CA. Mixing irreversible hydrocolloid with no bubbles or hassles. Journalof Prosthetic Dentistry 1992; 68: 558560.

[4] Oldman PA, Jemt TM. Accuracy of impression materials in a semi-clinical model. DentalMaterials 1988; 4: 64-67.

[5] Dhal BL, Dymbe B, Valdenhang J. Bonding properties and dimensional stability ofhydrocolloid impression systems in fixed prosthodontics. Journal of Prosthetic Dentistry 1985; 53: 795-800. 